\title{
Axonal degeneration and inflammation in acute optic neuritis
}

\author{
A Petzold, K Rejdak, G T Plant
}

J Neurol Neurosurg Psychiatry 2004;75:1178-1 180. doi: 10.1136/jnnp.2003.017236

Aims: To investigate whether plasma biomarkers for axonal injury and inflammation are related to loss and recovery of visual function in acute optic neuritis (ON).

Methods: Eighteen patients with $\mathrm{ON}$ and 14 controls were investigated in a longitudinal, prospective study. Plasma phosphorylated neurofilament heavy chain ( $\mathrm{NFH}^{\mathrm{SMI} 35}$; a surrogate marker of axonal injury), nitric oxide metabolites (NOx), and citrulline (surrogate markers of inflammation) were measured.

Results: Patients with $\mathrm{ON}$ had higher median plasma $\mathrm{NFH}^{S M / 35}$ values than controls $(0.17$ versus $0.005 \mathrm{ng} / \mathrm{ml}$; $\mathrm{p}<0.05$ ) and higher NOx values $(49$ versus $35.5 \mu \mathrm{M}$; $\mathrm{p}<0.001)$. Plasma $\mathrm{NFH}^{\mathrm{SMI} 35}$ values correlated inversely with visual acuity at presentation $(R=-0.67 ; p=0.01)$. $\mathrm{NFH}^{\mathrm{SM} / 35}$ was higher in patients with poor recovery of visual acuity than in those with good recovery $10.25 \mathrm{ng} / \mathrm{ml}$ versus $0.09 \mathrm{ng} / \mathrm{ml} ; \mathrm{p}<0.05)$. Three of four patients with high $\mathrm{NfH}^{\mathrm{SMl} 35}$ and high NOx values experienced a poor recovery as opposed to only one of five with high NOx but normal $\mathrm{NFH} \mathrm{SMI35}^{\mathrm{s}}$ values.

Conclusions: $\mathrm{NFH}^{\mathrm{SMI} 35}$, a surrogate marker for axonal damage, is a prognostic indicator and should be considered in the design of neuroprotective treatment strategies.

$\mathrm{T}$ he "axonal death cascade"1 has been associated with nitric oxide (NO) mediated damage in vitro. ${ }^{2}$ However, the relation of axonal degeneration to NO release in vivo is not known. Acute optic neuritis provides a useful model because of the defined onset and the anatomically restricted area of neuronal damage. ${ }^{3}$ Increased concentrations of the $\mathrm{NO}$ metabolites (NOx) nitrite $\left(\mathrm{NO}^{2-}\right)$ and nitrate $\left(\mathrm{NO}^{3-}\right)$ were found in the cerebrospinal fluid and serum of patients with optic neuritis (ON). ${ }^{56}$ Axonal loss appears to be linked to the acquisition of a permanent deficit. ${ }^{27-12}$ However, the role of inflammation in demyelinating disease is less well defined, and there is accumulating evidence that immune mediated inflammation has neuroprotective properties. ${ }^{13}$

Our study aimed to investigate the relation between the loss and recovery of optic nerve function with surrogate markers of inflammation (citrulline and the NO metabolites $\mathrm{NO}^{2-}$ and $\left.\mathrm{NO}^{3-}\right)^{214-16}$ and for axonal injury (neurofilaments ${ }^{17}$ ) in patients with acute optic neuritis.

\section{PATIENTS AND METHODS}

Patients presenting with symptoms suggestive of acute unilateral ON $(\mathrm{n}=18)$ and age matched healthy controls $(n=14)$ were investigated in this prospective study (table 1). Inclusion criteria for patients with ON were: progressive loss of vision over a few days, decreased visual acuity (VA; Snellen chart), decreased colour vision (Ishihara colour plates), and relative afferent pupillary defect. ${ }^{3}$ Because VA was severely affected in most cases, the visual fields were usually assessed by confrontation. Because this is not a quantitative method, this precluded the use of perimetry in the quantitative assessment. Exclusion criteria were: any other macular or retinal pathology, contemporaneous symptoms suggestive of demyelination elsewhere, or lack of spontaneous recovery. Our study was approved by the local ethics committee. Recovery of VA and colour vision was assessed at each visit and once recovery stabilised no further follow up was arranged. The last recorded VA was taken as the final outcome. Brain and optic nerve magnetic resonance imaging (MRI) was performed using a 1.5 Tesla system (Siemens AG, Erlangen, Germany) and consisted of axial Tl and T2 weighted spin echo MRI. ${ }^{35}$ Plasma was taken at the

Abbreviations: $M R L$, magnetic resonance imaging; $M S$, multiple sclerosis; $\mathrm{NFH}^{\mathrm{SM} 135}$, phosphorylated neurofilament heavy chain; NOx, nitric oxide metabolites; $\mathrm{ON}$, optic neuritis; VA, visual acuity

\begin{tabular}{llll} 
Table 1 & \multicolumn{3}{l}{ Characteristics of patients expressed as medians (interquartile range) } \\
\hline Feature & Controls & Acute optic neuritis & p Value \\
\hline Age (years) & $36(30-43)$ & $36(35-46)$ & \\
Male/female & $4 / 10$ & $9 / 5$ & $<0.05$ \\
Eye & $\mathrm{N} / \mathrm{A}$ & $\mathrm{R} 8 / \mathrm{L} 6$ & \\
RAPD & $\mathrm{N} / \mathrm{A}$ & $\mathrm{R} 8 / \mathrm{L} 5$ & \\
Visual acuity & 1.2 & $0.2(0.05-0.67)$ & \\
Colour vision & $17 / 17$ & $0 / 17(0 / 17-7 / 17)$ & \\
Disc appearance & $\mathrm{Normal}$ & 2 swollen, 12 normal & \\
Time from onset & $\mathrm{N} / \mathrm{A}$ & 14 days $(8-26)$ & \\
Follow up & $\mathrm{N} / \mathrm{A}$ & 171 days $(25-189)$ & \\
MRI & $\mathrm{N} / \mathrm{A}$ & 10 & $<0.001$ \\
Plasma NfH SMI35 $(\mathrm{ng} / \mathrm{ml})$ & $0.005(0.0-0.094)$ & $0.17(0.07-0.33)$ & $<0.01$ \\
Plasma NOx $(\mu M)$ & $36.2(31.2-38.6)$ & $48.1(40.8-54.2)$ & $\mathrm{NS}$ \\
Plasma citrulline $(\mathrm{nmol} / \mathrm{ml})$ & $83.4(72.1-92.4)$ & $67.9(62.5-91.9)$ & \\
\end{tabular}

$\mathrm{MRl}$, magnetic resonance imaging; $\mathrm{NA}$, not assessed; $\mathrm{NFH}^{\mathrm{SM} 135}$, phosphorylated neurofilament heary chain; nitric oxide metabolites; RAPD, relative afferent pupillary deficit; VFD, visual field defect. 
first visit and concentrations of the phosphorylated neurofilament heavy chain $\left(\mathrm{NfH}^{\mathrm{SMI} 35}\right), \mathrm{NOx}$, and citrulline were measured as described previously. ${ }^{14} 1617$

Data analysis was performed using SAS. Because of nonGaussian data distribution the medians and interquartile ranges are shown. All correlations were studied using Spearman's rank correlation coefficient. Differences between groups were compared using the two sided Wilcoxon two sample test. Significances based on small numbers were checked on a categorical level using the two sided Fischer's exact test. Trend analysis was carried out using the MantelHaenzel $\chi^{2}$ test. A $5 \%$ level of significance was used throughout.

\section{RESULTS}

The diagnosis of ON was confirmed in all 18 patients during follow up. However, in four patients the blood sample was taken over four weeks after onset of symptoms. Therefore, these patients were excluded from our analysis. The remaining 14 patients presented with a median time from onset of two weeks (table 1). At presentation, 10 of 14 of the patients had a VA of less then $0.33(6 / 18,20 / 60$ Snellen equivalent). In total, 13 of 14 patients showed sustained improvement of their VA during follow up. A minor degree of optic atrophy was seen in four of nine patients whose VA recovered to over $0.33(6 / 18)$. Optic nerve atrophy was more severe in those five of the patients who did not recover their VA above 0.33 .

Plasma concentrations of $\mathrm{NfH}^{\mathrm{SMI} 35}$ (fig $\mathrm{l}$ A) and $\mathrm{NOx}$ (fig IB) were significantly higher in patients with ON than in controls ( $p<0.001$ and $p<0.01$, respectively). No such difference was found for citrulline (table 1). There was a negative correlation between $\mathrm{NfH}^{\mathrm{SMI} 35}$ and VA at the time of first presentation $(R=-0.67 ; \mathrm{p}=0.0 \mathrm{l}$; fig $\mathrm{lC})$. One outlier with $\mathrm{NfH}^{\text {SMI35 }}$ values of $0.65 \mathrm{ng} / \mathrm{ml}$ was seen. This 48 old male patient presented with a right ON, VA was reduced to hand movement $(6 / 60,20 / 200$ Snellen equivalent), colour vision to $0 / 17$, and there was a right relative afferent pupillary deficit. This patient had suffered from an episode of myelitis two years previously. In the absence of clinical signs of myelitis, no spinal MRI was performed. After the removal of this patient from our study the correlation between $\mathrm{NfH}^{\mathrm{SMI} 35}$ and VA remained significant $(R=-0.60$; $\mathrm{p}<0.05)$, but the slope of the linear regression decreased from -0.22 to -0.145 .

No correlation between VA and either NOx or citrulline was found at presentation. There was no correlation between $\mathrm{NfH}^{\mathrm{SMI35}}, \mathrm{NOx}$, or citrulline and age or time from onset; in addition, there were no sex differences.

Plasma $\mathrm{NfH}^{\mathrm{SMI} 35}$ concentrations at presentation were significantly higher (mean, $0.25 \mathrm{ng} / \mathrm{ml}$ ) in the patients with poor recovery of visual function $(0.33 ; 6 / 18,20 / 60$ Snellen equivalent) when compared with those whose VA recovered to above 0.33 (mean, $0.09 \mathrm{ng} / \mathrm{ml} ; \mathrm{p} \leqslant 0.05$ ). The difference remained significant when comparing proportions of patients with $\mathrm{NfH}^{\mathrm{SMI35}}$ values above the cut off value (fig 1A; $p=0.015$; two sided Fisher's exact test). There were no differences in NOx values at presentation between those with good versus poor visual recovery at follow up (54.2 versus $44.6 \mu \mathrm{M} ; \mathrm{p}=0.1$ ).

The combined association between raised NOx and $\mathrm{NfH}^{\mathrm{SMI35}}$ concentrations and recovery of visual function was investigated by comparing proportions of patients with high values (raised above the top value of the control group; $\left.\mathrm{NfH}^{\mathrm{SMI} 35},>0.17 \mathrm{ng} / \mathrm{ml} ; \mathrm{NOx},>43.8 \mu \mathrm{M}\right)$. There was a significant trend for increased proportion of patients experiencing a poor recovery (Mantel-Haenzel $\chi^{2}=4.6 ; p<0.05$ ) across the following categories: $\mathrm{NfH}^{\mathrm{SMI} 35}$ normal and NOx normal (none of three, $0 \%$ poor recovery), $\mathrm{NfH}^{\mathrm{SMI} 35}$ normal and NOx high (one of five, $20 \%$ poor recovery), $\mathrm{NfH}^{\mathrm{SMI} 35}$
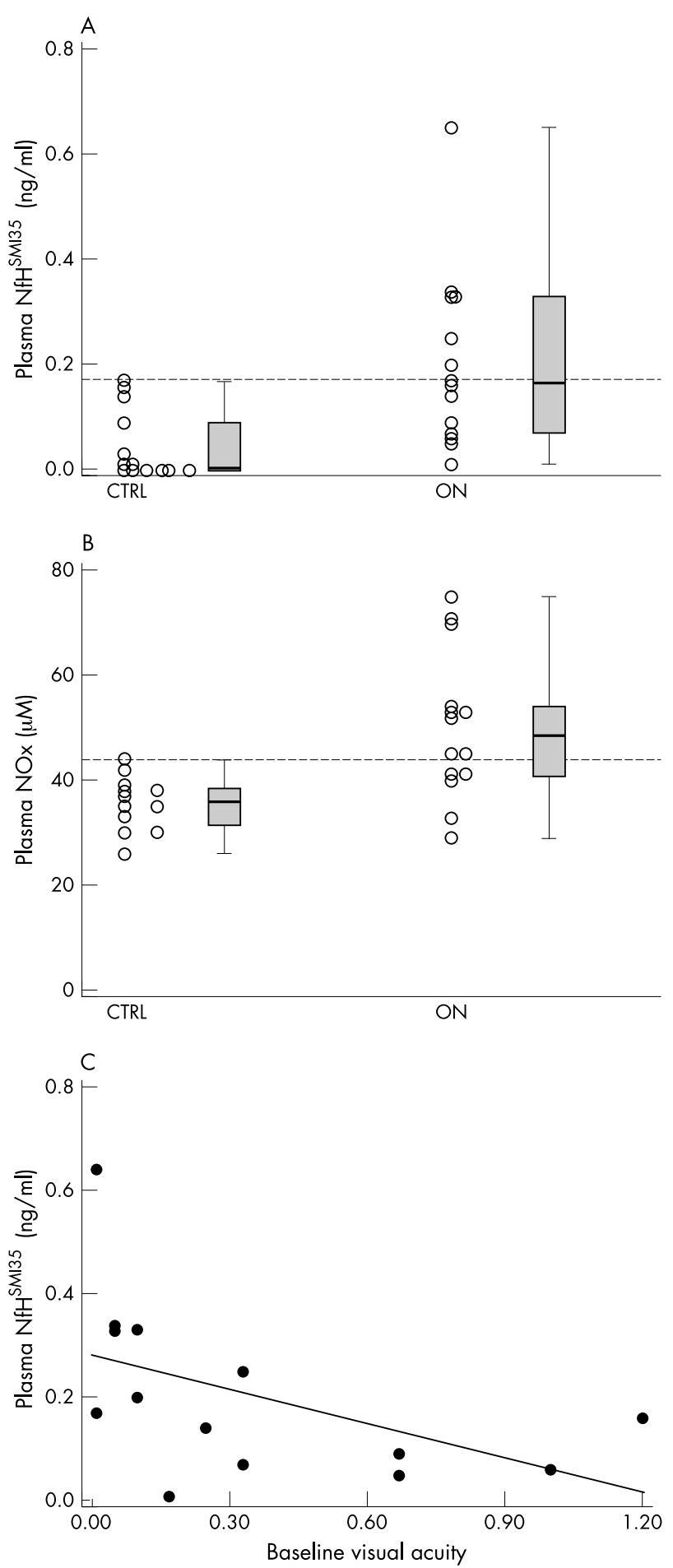

Figure 1 (A) Plasma phosphorylated neurofilament heavy chain $\left(\mathrm{NfH}^{\mathrm{SMl} 35} ; \mathrm{ng} / \mathrm{ml}\right.$ ) concentrations and (B) nitric oxide metabolites (NOx; $\mu \mathrm{M}$ ) concentrations in controls (CTRL) and patients with acute optic neuritis $(\mathrm{ON})$. The median $(50 \%)$, box $(25-75 \%)$, and whisker $(0 \%-$ $100 \%$ ) values are shown next to the individual values (open circles). (C) Plasma NfH ${ }^{\mathrm{SM} 135}$ correlates with visual acuity at the time of sampling $(R=-0.66 ; p=0.01)$.

high and NOx normal (one of two, 50\% poor recovery), $\mathrm{NfH}^{\mathrm{SMI} 35}$ high and NOx high (three of four, $75 \%$ poor recovery).

MRI results were available in 10 of 14 patients; six had an isolated ON lesion (mean $\mathrm{NfH}^{\mathrm{SMI} 35}, 0.12 \mathrm{ng} / \mathrm{ml}$; mean NOx, $40.7 \mu \mathrm{M})$, as opposed to four with disseminated brain lesions 


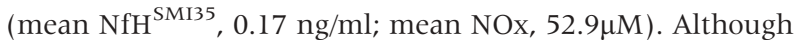
$\mathrm{NfH}^{\mathrm{SMI} 35}$ and NOx seemed to be marginally higher in patients with disseminated brain lesions this did not reach significance.

\section{DISCUSSION}

Ours is the first study to measure biomarkers for axonal injury ( $\mathrm{NfH}^{\mathrm{SMI} 35}$ ) and inflammation (NOx, citrulline) in the plasma of patients with acute ON. The main findings were that $\mathrm{NfH}^{\mathrm{SMI35}}$ concentrations were higher in patients with ON than in controls, they were inversely correlated with VA at presentation, and they were of prognostic relevance. This suggests the presence of axonal damage in the acute phase. The results are in line with clinical, MRI, and electrophysiological evidence of optic nerve fibre loss following a single episode of ON and insidiously in multiple sclerosis (MS)..$^{18-20}$

Recovery of the VA to $>6 / 18(20 / 60)$ was achieved by fewer patients $(36 \%)$ than recovery of the VA to $>20 / 50$ $(88 \%)$ in the optic neuritis treatment trial after five years of follow up. ${ }^{4}$ Bradley and Whitty (1967) found that recovery of VA to $>20 / 30$ was achieved by $50 \%$ of patients after one month and $75 \%$ after six months. ${ }^{21}$ The results of our present study may relate to the small sample size and the relatively short period of follow up (25-189 days).

Recovery of VA in MS related ON has been attributed to resolution of the conduction block caused by acute inflammation. ${ }^{19}{ }^{20} \mathrm{NO}$ mediated conduction block also seems to be the initial mechanism in in vitro studies. ${ }^{2223}$ Our present study and one other study 5 failed to show a significant relation between serum NOx values and outcome. In addition, NOx and $\mathrm{NfH}^{\mathrm{SMI} 35}$ did not correlate with MRI parameters, but this could be a result of the small study population. The almost $30-40 \%$ higher values, if true, might indicate that demyelinating activity elsewhere is contributing to the worse outcome.

Citrulline is thought to be another marker of NO mediated inflammation, but it did not correlate with NOx and no differences were seen between patients with $\mathrm{ON}$ and controls. This finding is in keeping with one study comparing cerebrospinal fluid and serum citrulline concentrations between patients with Lewy body dementia and healthy controls. ${ }^{18}$ We agree with these authors that citrulline is not a good surrogate marker for NO metabolism because it is also a substrate for enzymes other than inducible nitrogen oxide synthetase.

In MS, it appears that inflammatory markers are predominately related to disease activity and the MRI lesion load but not to outcome. ${ }^{54}$ In addition, epidemiological data suggest that inflammation in MS has only a limited effect on the course of neurodegeneration. ${ }^{25}$ In placebo controlled trials and clinical experience with $\mathrm{ON}$, anti-inflammatory treatment with steroids does not influence outcome in most cases. ${ }^{3}$ The destructive and neuroprotective aspects of inflammation have stimulated a controversial discussion. ${ }^{15}{ }^{13} 25-27$ Therefore, it is interesting that in this small group, four of five of the patients with raised NOx concentrations but normal $\mathrm{NfH}^{\mathrm{SMI} 35}$ values made a good recovery. This finding highlights the potential for selection bias in treatment trials for neuroprotective drugs by inclusion of those patients with evidence of inflammation only.

Taken together, these findings support the concept that sustained loss of optic nerve function relates to axonal degeneration and that NO might contribute, at least in part, to the "axonal death cascade". ${ }^{2}{ }^{27-9}$

\section{ACKNOWLEDGEMENTS}

Dr K Rejdak was supported by The Foundation for Polish Science (FNP).

\section{Authors' affiliations}

A Petzold, Department of Neuroinflammation, Institute of Neurology, University College London, Queen Square, London WCIN 3BG, UK G T Plant, Moorfields Eye Hospital, Department of Neuroophthalmology, City Road, London ECJ 2PD, UK K Rejdak, Department of Neurology, Medical University of Lublin, Poland

Competing interests: none declared

Correspondence to: Dr A Petzold, Department of Neuroinflammation, Institute of Neurology, Queen Square, London WCIN 3BG, UK; a.petzold@ion.ucl.ac.uk

Received 2 May 2003

In revised form 27 October 2003

Accepted 28 October 2003

\section{REFERENCES}

1 Waxman S. Nitric oxide and the axonal death cascade. Ann Neurol 2003:53:150-3.

2 Kapoor R, Davies M, Blaker P, et al. Blockers of sodium and calcium entry protect axons from nitric oxide-mediated degeneration. Ann Neurol 2003;53:174-80

3 Hickman S, Dalton C, Miller D, et al. Management of acute optic neuritis. Lancet 2002;360:1953-62.

4 Beck R. Optic neuritis. In: Miller NR, ed. Walsh and Hoyt's clinical neuroophthalmology, 5th ed. Lippincott, Williams and Wilkins, 1998:599-647.

5 Giovannoni G, Miller D, Losseff N, et al. Serum inflammatory markers and clinical MRI markers of disease progression in multiple sclerosis. J Neurol $2001 ; 248: 487-95$.

6 Sellebjerg F, Giovannoni G, Hand A, et al. Cerebrospinal fluid levels of nitric oxide metabolites predict response to methylprednisolone treatment in multiple sclerosis and optic neuritis. J Neuroimmunol 2002;125:198-203.

7 Frisen L, Hoyt W. Insidious atrophy of retinal nerve fibers in multiple sclerosis. Funduscopic identification in patients with and without visual complaints. Arch Ophthalmol 1974;92:91-7.

8 Hickman S, Kapoor R, Jones S, et al. Corticosteroids do not prevent optic nerve atrophy following optic neuritis. J Neurol Neurosurg Psychiatry 2003;74:1139-41.

9 Hickman S, Brierley C, Brex P. Continuing optic nerve atrophy following optic neuritis: a serial MRI study. Mult Scler 2002;8:339-42.

10 Trapp B, Peterson I, et al. Axonal transection in the lesions of multiple sclerosis. N Engl J Med 1998;338:278-85.

11 Losseff N, Webb S, O'Riordan J, et al. Spinal cord atrophy and disability in multiple sclerosis: a new reproducible and sensitive MRI method with potential to monitor disease progression. Brain 1996;119:701-8.

12 Waxman S. Demyelinating diseases - new pathological insights, new therapeutic targets. N Engl J Med 1998;338:323-5.

13 Nguyen M, Julien J, Rivest S. Innate immunity: the missing link in neuroprotection and neurodegeneration? Nat Rev Neurosci 2002;3:216-27.

14 Alderton W, Cooper C, Knowles R. Nitric oxide synthases: structure, function and inhibition. Biochem J 2001;357:593-615.

15 Kamoun P, Parvy P, Cathelineau L. Rapid and simple method for citrulline determination in plasma. Clin Chem 1983;29:398-400.

16 Miranda K, Espey M, Wink D. A rapid, simple spectrophotometric method for simultaneous detection of nitrate and nitrite. Nitric Oxide 2001;5:62-71.

17 Petzold A, Keir G, Green A, et al. A specific ELISA for measuring neurofilament heavy chain phosphoforms. J Immunol Methods 2003;278: 179-90.

18 Molina J, Leza I, Ortiz S, et al. Cerebrospinal fluid and plasma concentrations of nitric oxide metabolites are increased in dementia with Lewy bodies. Neurosci Lett 2002;333:151-3.

19 Brusa A, Jones S, Plant G. Long-term remyelination after optic neuritis: a 2 -year visual evoked potential and psychophysical serial study. Brain 2001;124:468-79.

20 Youl B, Turano G, Miller D, et al. The pathophysiology of acute optic neuritis and association of gadolinium leakage with clinical and electrophysiological deficits. Brain 1991;114:2437-50.

21 Bradley W, Whitty C. Acute optic neuritis: its clinical features and their relation to prognosis for recovery of vision. J Neurol Neurosurg Psychiatry 1967;30:531-8.

22 Smith K, Hall S. Factors directly affecting impulse transmission in inflammatory demyelinating disease: recent advances in our understanding. Curr Opin Neurol 2001;14:289-98.

23 Smith K, Kapoor R, Hall S, et al. Electrically active axons degenerate when exposed to nitric oxide. Ann Neurol 2001;49:470-6.

24 Acar $G$, Idiman $F$, Idiman $E$, et al. Nitric oxide as an activity marker in multiple sclerosis. J Neurol 2003;250:588-92.

25 Confavreux C, Vukusic S, Adeleine P. Early clinical predictors and progression of irreversible disability in multiple sclerosis: an amnesic process. Brain 2003; 126:770-82.

26 Confavreux C, Vukusic S, Moreau T, et al. Relapses and progression of disability in multiple sclerosis. N Engl J Med 2000;343:1430-8.

27 Rieckmann P, Smith K. Multiple sclerosis: more than inflammation and demyelination. Trends Neurosci 2001;24:435-7. 